ISSN: 2550 - 682X

DOI: $10.23857 /$ pc.v3i11.777

Recepción: 22 / 07 / 2018

Aceptación: 17 / 09 / 2018

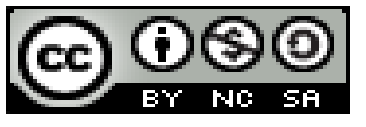

Ciencias sociales y políticas

Artículo de revisión

\title{
El concepto metáfora paterna y su relación con conductas sociales
}

desajustadas

\section{The paterna metaphor concept and its relationship with unsetted social behaviors O conceito do metáfor de paterna e sua relação com os comportamentos sociais não-confessados}

\author{
Amira G. Trujillo-Eljuri ${ }^{\mathrm{I}}$ \\ amiratrujillo@uleam.edu.ec \\ Inés G. Vera-Molina II \\ amira.trujillo@uleam.edu.ec \\ María A. Carvajal-García ${ }^{\text {III }}$ \\ e1312302035@live.uleam.edu.ec
}

María F. Solórzano-Muente IV

e1313602771@live.uleam.edu.ec

Melissa B. Tóala-Plúa V

e1316284122@live.uleam.edu.ec

Vanessa G. Villafuerte-Merchán VI

e1316263134@live.uleam.edu.ec

Correspondencia: ines.vera@uleam.edu.ec

\footnotetext{
${ }^{\text {I } M a g i ́ s t e r ~ e n ~ E d u c a c i o ́ n ~ S u p e r i o r, ~ P s i c o ́ l o g o ~ C l i ́ n i c o, ~ D o c e n t e ~ d e ~ l a ~ U n i v e r s i d a d ~ L a i c a ~ E l o y ~ A l f a r o ~ d e ~ M a n a b i ́, ~}$ Manta, Ecuador.

${ }^{\text {II } M a g i ́ s t e r ~ e n ~ P s i c o a n a ́ l i s i s ~ c o n ~ M e n c i o ́ n ~ e n ~ C l i ́ n i c a ~ P s i c o a n a l i ́ t i c a, ~ P s i c o ́ l o g a ~ C l i ́ n i c a, ~ D o c e n t e ~ d e ~ l a ~ U n i v e r s i d a d ~}$ Laica Eloy Alfaro de Manabí, Manta, Ecuador.

III Estudiante de la Facultad de Psicología de la Universidad Laica Eloy Alfaro de Manabí, Manta, Ecuador.

IV Estudiante de la Facultad de Psicología de la Universidad Laica Eloy Alfaro de Manabí, Manta, Ecuador

${ }^{\vee}$ Estudiante de la Facultad de Psicología de la Universidad Laica Eloy Alfaro de Manabí, Manta, Ecuador.

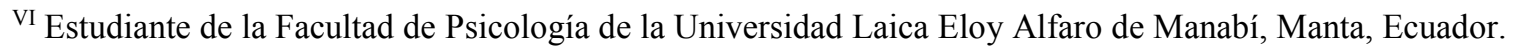




\section{Resumen}

No podríamos departir la conceptualización sobre la metáfora paterna sin las concepciones de Sigmund Freud, el cual cita las características esenciales para la manifestación de la función del padre, seguido de la expresión de los tiempos del padre definidos por Jacques Lacan. En tal sentido, se presenta en este artículo la caracterización del concepto metáfora paterna y su relación con conductas sociales desajustadas. La metodología siguió un enfoque cualitativo hermenéutico. Para la realización de la revisión sistemática se llevó una orden y coherencia sobre el tema del concepto de metáfora paterna y conductas sociales desajustadas y se hizo en tres momentos; en el primer momento se establecieron como criterios del estudio la identificación de los diseños admisibles para su revisión sistemática, en el segundo momento se hizo una codificación de los estudios que cumplieron con los criterios para el estudio, y en el tercer momento se realizó un análisis de la información, que permitió generar los resultados del estudio que permitieron conocer el manejo del concepto de Metáfora paterna y su relación con las conductas sociales desajustadas, de la que se pudo concluir que las conductas desajustadas en los adolescentes son conductas que se van desarrollando a lo largo de la vida de los mismos, así que estas son producto de cómo se va estructurando su Yo y superyó y asimismo de su estructura clínica y aquello que lo conforman.

Palabras clave: metáfora paterna; conducta desajustada; adolescente; agresión.

\section{Abstract}

We could not socialize the conceptualization of the paternal metaphor without the ideas of Sigmund Freud, which cites the essential features for the manifestation of the role of the father, followed by the expression of the father times defined by Jacques Lacan. In this sense, is presented in this article characterization of the concept paternal metaphor and its relation with mismatched social behaviors. The methodology followed a qualitative-hermeneutic approach. For the realization of the systematic review was an order and coherence on the subject of the concept of paternal metaphor and mismatched social behavior and was done in three stages; the first time were established as criteria of the study identification of eligible designs for systematic review, at the second time was a codification of the studies that met the criteria for the study, and in the third moment is an analysis of the information, allowing you to produce the results of the study that allowed to know the handling of the concept of paternal metaphor and its relation with the 
mismatched social behaviors, which could be concluded that conduct wrongly in adolescents are behaviors that will develop over the life of the same, so these are how product takes shape his ego and superego and also its clinical structure and what is made up.

Key words: paternal metaphor; maladjusted; adolescent behavior; aggression.

\section{Resumo}

Nós não poderíamos socializar a conceituação da metáfora paterna, sem as idéias de Sigmund Freud, que cita as características essenciais para a manifestação do papel do pai, seguido da expressão dos tempos definidos por Jacques Lacan pai. Nesse sentido, a caracterização do conceito de metáfora paterna e sua relação com comportamentos sociais desajustados é apresentada neste artigo. A metodologia seguiu uma abordagem hermenêtica qualitativa. Para realizar a revisão sistemática, foi realizada uma ordem e coerência no tema do conceito de metáfora paterna e comportamentos de desadaptação social e foi realizada em três momentos; na primeira foram estabelecidos como critérios do estudo identificação dos projetos elegíveis para a sua revisão sistemática, no segundo tempo de codificação estudos que preencheram os critérios para o estudo foi feito, ea análise terceira vez foi realizada informações, o que permitiu gerar os resultados do estudo permitiu conhecer o conceito de gestão da metáfora paterna e sua relação com o comportamento social desajustado, a partir do qual concluiu-se que a conduta desajustados em adolescentes são comportamentos que são desenvolvidos para ao longo de suas vidas, então estes são um produto de como eles estão estruturando seu ego e superego e também de sua estrutura clínica e do que eles são feitos.

Palavras chave: metáfora paterna; comportamento incompatível; adolescente agressão.

\section{Introducción}

En la actualidad son numerosos los casos de adolescentes con problemas de comportamiento tanto en el ámbito familiar como el educativo, por años se ha definido a estos problemas como conductas desajustadas, es así que, dentro del grupo de investigación de psicoanálisis de la facultad de psicología de la ULEAM, ha nacido la curiosidad de cuál es el origen de estas conductas, presentándose la hipótesis de que su origen se relaciona con el declive de la metáfora 
paterna. En la presente revisión bibliográfica se busca relacionar el declive de la metáfora paterna con las conductas desadaptadas, tomando como grupo de estudio a los adolescentes, basando en la literatura de destacados psicoanalistas como Sigmund Freud y Jacques Lacan, todo esto con el objetivo de identificar las características de esto jóvenes y corroborar la hipótesis, para así posteriormente poder desenredar ese nudo en su estructura.

Este trabajo se propone definir la función paterna para el psicoanálisis, la misma se deduce de los trabajos de Sigmund Freud y de las primeras elaboraciones de Lacan sobre dichos trabajos, dado que en los desarrollos posteriores sobre la función paterna es posible reconocer las marcas de este primer momento conceptual.

La función paterna, expresión introducida por Lacan, se desprende de la elaboración del Complejo de Edipo por parte de Sigmund Freud y de los desarrollos de Jacques Lacan que toman esta como punto de partida. Para esto Lacan lee el Edipo freudiano desde la lingüística estructural aplicando a éste la teoría del significante, el resultado es haber descubierto la estructura de lenguaje que subyace a la teoría freudiana sobre el padre y que él formaliza a través de la metáfora paterna, primera elaboración de Lacan en torno al padre, cuyo poder Complejo de Edipo del Seminario 17 inaugural dejará marcas indelebles en el concepto de dicha función.

Tal como dice Lacan en el capítulo Más allá del Complejo de Edipo del Seminario 17 El Reverso del Psicoanálisis, su modo de abordar la cuestión del Edipo fue la introducción de la metáfora paterna: (...) fue en este nivel como hablé de la metáfora paterna. Del complejo de Edipo no hablé nunca más que de esta forma. (...) Dije que se trataba de la metáfora paterna, mientras que sin embargo no es así como Freud nos presenta las cosas. Bajo esta concepción se presenta en este artículo una caracterización del concepto metáfora paterna y su relación con conductas sociales desajustadas.

\section{Desarrollo}

La función del padre desde Freud y el Complejo de Edipo según Lacan. Para poder describir la función paterna debemos revisar el complejo de Edipo desde Sigmund Freud, debido a que éste tiene un papel central puesto que se da la entrada a la sociedad y las características ya sean viriles o femeninas del sujeto, es esencial para que se dé la función del padre, ya sea real, imaginario o simbólico puesto que sin una función paterna no hay Edipo. 
El Edipo de Jacques Lacan (1958) se divide en tres tiempos; son tiempos lógicos, no cronológicos, que nos ayudan a pensar la clínica y la constitución del sujeto. Tiempo lógico porque depende del momento psíquico en el que se encuentra el sujeto mas no en cuanto al tiempo cronológico que implica el proceso evolutivo en el que se encuentra el niño(a).

En el primer tiempo del Edipo, el niño desea ser el objeto de deseo de la madre, en el segundo interviene el padre en tanto que a cumplir su función de la que hablaremos más adelante, a privar al niño de la madre la cual es su objeto de deseo, por lo que la madre dejaría de ser fálica y el niño deja de ser el falo (castración simbólica); punto importante de indicar que es siempre de doble vía tanto para la madre como para el hijo; pero entonces el padre dejaría de ser la ley es ahí cuando se da el tercer tiempo del Edipo en el que el padre debe reconocer que también está en falta. La ley quedará instaurada por la sociedad, la cultura como falo simbólico. En este tercer tiempo del Edipo, el falo y la ley quedan instaurados como instancias que están más allá de cualquier personaje (Bleichmar, 1980)

En el Edipo existen fantasmas que hacen el intento de solución a esas incógnitas que le surgen a los seres humanos y que son las preguntas sobre su sexualidad (fantasma de seducción), la diferencia de los sexos (fantasma de castración) y su origen (escena originaria). Estos fantasmas son las representaciones de la posición del sujeto en el discurso en relación al objeto de su deseo.

¿Qué es un padre? Varón o animal macho que ha engendrado a otro ser de su misma especie, ejerce las funciones de padre, destinado a la procreación, por ende, jefe de una descendencia, familia o pueblo. Desde el psicoanálisis, un padre es el que cumple una función, es un nombre, el padre es quién edifica y organiza la realidad anímica, el padre, aunque no esté presente físicamente va a existir ya que es la madre quién le da ese nombre al padre, él puede existir incluso sin estar presente, él prohíbe a la madre y es ella quién le da el poder y la ley al padre frente al hijo.

Freud en su estudio con sus histéricas como él las denominaba, comienza a desplegar la teoría de la seducción, la cual en su desarrollo perdió crédito. Esto debido a que en todos los casos el padre debía ser culpado como perverso y Freud comenzó a dudar de esto, lo que lo llevó a su descredito de la Teoría de la Seducción y su apego a la incipiente Teoría del Edipo. (Freud, 1899/1996) 
Para el varón el padre y para la niña las madres devinieron competidores estorbosos, y ya respecto de los hermanos puntualizaremos cuán poco se necesita para que este sentimiento lleve al deseo de muerte. Es regla que la preferencia sexual se imponga ya en los propios padres; un impulso natural vela porque el hombre alague a su pequeña y la madre favorezca al varón, al paso que ambos, donde el ensalmo del sexo no enturbia su juicio, se empeña con rigor en la educación de sus hijos. El niño advierte muy bien la preferencia y se revuelve contra el miembro de la pareja parental que se le opone. Para él, hallar amor en el adulto no es sólo la satisfacción de una necesidad particular; también significa que su voluntad será obedecida en todo lo demás. Así, cuando elige a uno de sus progenitores en el mismo sentido en que ellos lo hacen, cede a su propia pulsión sexual, renovando al mismo tiempo la incitación que partió de ellos. (Freud, 19001901/1996, p.267)

De modo analítico el Complejo de Edipo, según Laplanche y Pontalis (2010) indican que Freud utiliza el término Complejo de Edipo en el siguiente sentido: Conjunto organizado de representaciones y de recuerdos dotados de intenso valor afectivo, parcial o totalmente inconscientes. Un complejo se forma a partir de las relaciones interpersonales de la historia infantil; puede estructurar todos los niveles psicológicos: emociones, actitudes, conductas adaptadas. (p.55)

La metáfora paterna concierne a la función del padre (...) La función del padre tiene su lugar, un lugar bastante amplio, en la historia del análisis. Se encuentra en el corazón de la cuestión del Edipo y ahí es donde la ven ustedes personificada. Freud la introdujo al principio de todo, porque el complejo de Edipo aparece ya de entrada en La interpretación de los sueños (Lacan, 195758/2007, p. 165)

La declinación del Padre. Han pasado décadas desde que Sigmund Freud y Jacques Lacan, realizaron las conceptualizaciones teóricas del Complejo de Edipo y metáfora paterna, correspondientemente. Sigmund Freud aborda al padre como agente de seducción, objeto de pulsiones, función de prohibición del incesto y como dimensión existencial, por su parte Lacan considera al padre como la internalización de las estructuras sociales, mediando al objeto por la cultura y el lenguaje, que reproduce cierta realidad del ambiente (La familia, Lacan, 1938/1978), pero se pregunta será que en la actualidad se sigue presenciando esa misma metáfora paterna, ya 
que como bien se sabe los roles dentro de la familia han sufrido grandes cambios, o será que este declinar tiene un aspecto mayormente social?

Se conoce desde la teoría psicoanalítica, según Uribe (2010), que la primera identificación del sujeto se da en el estadio del espejo, dando paso a la conformación de una instancia psíquica del superyó -con su voz femenina haciendo cumplir las reglas-, el ingreso a la cultura y así mismo dando lugar al significante del padre y a la ya conocida metáfora paterna o metáfora de ley, entonces el adolescente que experimente la forclusión en este tiempo no solo tendrá un rechazo por el padre sino un rechazo de la sociedad, se podría pensar que es esto lo que provoca una conducta desajustada, que es una protesta a las reglas de la misma, una protesta a lo que le fue negado.

La pubertad y adolescencia es una etapa de crisis pero que hace diferente a esta crisis, si a lo largo de nuestra vida desde nuestro nacimiento hasta nuestra muerte están presentes las mismas, según E. Erikson "la identidad yoica se sustenta en procesos de identificación que en principio se refieren a las figuras paternas, pero que luego se vinculan con otros objetos exogámicos, que sin embargo se perfilan como sustitutos de los primeros", he aquí mi cuestionamiento que sucede con los púberes o adolescentes que no pasaron por esa primera identificación? Existirán problemas y si los hay, ¿cuál es la razón? Se considera pues que lo que influye es la necesidad de identificación que se tiene en ella, es por ello que cuando no se cuenta con la principal identificación, la paterna, existe esa necesidad de suplencia con otras identificaciones, dentro de ellas típicamente se encuentran las de los grupos sociales independientemente sean estos positivos o negativos, ya que le brinda al adolescente lo que el necesita -la aceptación-.

Los cambios producidos en el cuerpo del púber tanto física como psicológica genera en él extrañeza, para Negro (2012), es un proceso de cambio pero también de aceptación a un YO nuevo, algo desconocido y es este mismo desconocimiento que permite que el síntoma se instaure en el cuerpo porque no puede explicarse por medio de la palabra, si nos situamos en los tatuajes o piercings de los miembros de pandillas se evidencia lo anteriormente mencionado y se explica mejor si se considera lo señalado por Medeiros, Silveira y Machado(2009) "es evidente que la intensidad de las excitaciones puede ser aumentada aún más por el fracaso de la elaboración psíquica por una falta de recursos de simbolización durante este período" 
Jacques Lacan en su Seminario III "Las Psicosis", nos ayuda a entender cómo se desarrolló la función paterna, utilizando un símil ilustrativo para explicarlo:

Puede que al comienzo el taburete no tenga suficientes pies, pero que igual se sostenga hasta cierto momento, cuando el sujeto, en determinada encrucijada de su historia biográfica, confronta ese defecto que existe desde siempre. Para designarlo nos hemos contentado por el momento con el término de Verwerfung (Lacan, 1956/2009, p.289)

Pero hay que señalar que el significante que estructura al sujeto no es cualquier significante, es el significante del-nombre-del-padre. Para que se produzca una psicosis, será necesario que falte la falta. Es decir que no se encuentre con la castración. El sujeto entraría en un plano imaginario y no en la tríada de Real, Simbólico e Imaginario, sino en una dialéctica, pero es necesario mencionar que para que esto suceda no solo depende de la falta real del padre, ya que si no se cumple la función de padre y si la madre no da el lugar que corresponde a pesar de que este físicamente ahí al sujeto le faltará la falta, no se topará con la castración, pero por el contrario si el padre muriera puede seguir cumpliendo esa función, si a través del discurso de la madre se lo ubica en el lugar que le corresponde, es decir en el lugar del sujeto deseante. Para J. Lacan, la Psicosis "se desencadena cuando desde el Otro hay un llamado de ese significante primordial que no puede ser aceptado."

\section{Metodología}

Se siguió una metodología con un enfoque cualitativo hermenéutico. Para la realización de la revisión sistemática en la cual es importante llevar un orden y coherencia sobre el tema del concepto de metáfora paterna y conductas sociales desajustadas se hizo en tres momentos; en el primer momento se establecieron como criterios del estudio la identificación de los diseños admisibles para su revisión sistemática, los tipos de programas, los tratamientos o intervenciones que se pretendían investigar, se definieron las características de las temáticas a revisar para el estudio y el idioma en el que debía estar escrito el estudio. Luego se procedió a realizar la búsqueda bibliografía lo más ampliamente posible, donde se incluyeron las consultas de bases bibliográficas electrónicas, otras fuentes fueron, la consulta directa de revistas especialmente sensibles al problema estudiado, así como la revisión de las referencias de los estudios que se localizaron, se contactaron expertos de reconocido prestigio en el campo quienes 
aportaron sus opiniones y se consultaron libros de congresos, tesis doctorales y otras estrategias que permitan acceder a la literatura fugitiva.

En el segundo momento se hizo una codificación de los estudios que cumplieron con los criterios para el estudio, y se procedió a elaborar un Manual de Codificación en el que se hicieron explícitos los criterios mediante los cuales se irían a codificar las características de los estudios. En esta fase se da la valoración de la calidad metodológica de los estudios empíricos.

En el tercer momento se realizó un análisis de la información, que permitió generar los resultados del estudio que permitieron conocer el manejo del concepto de Metáfora paterna y su relación con las conductas sociales desajustadas y así finalmente presentar las conclusiones del estudio.

\section{Análisis documental}

Para empezar a abordar el tema de una conducta desajustada, se hace necesario conocer las teorías acerca de las conductas desajustadas y que implica ésta para que sea denominada como tal.

La conducta se utiliza como medida de la personalidad humana de tal forma que somos lo que hacemos. Si la conducta empieza a tornarse desadaptada, esta sobrepasa los límites de la normalidad establecidos para vivir o convivir en sociedad y afecta al ámbito personal, familiar y social del individuo.

Hill (2003), refiere acerca de los problemas de conducta que son independientemente de las circunstancias sociales o familiares asociadas, predictores de una personalidad antisocial. Podríamos decir que los problemas de conducta son habituales en el entorno escolar y en muchos casos tienen consecuencias negativas graves, tanto para el alumno que se comporta inadecuadamente, como para el resto del grupo.

Dentro del enfoque psicoanalítico la perversión como tal se encuentra de una manera amplia, esta se visualiza de manera vasta y variada en respecto a lo fenomenológico.

En cuanto al tema oponiendo la "perversión verdadera" a las manifestaciones perversas episódicas de pacientes neuróticos o psicóticos, estos se muestran como grandes constantes las cuales configuran una estructura perversa, asimismo la relación específica del acto perverso con las angustias consideradas como confusionales, así también precisamente con el intento de los 
rituales considerados de una fantasía confusa de la escena primaria el desafío al padre y la profanación de la madre, las identificaciones bisexuales y confusas, el juego con la idea del incesto y la desmentida de la castración, la predominancia de elementos narcisistas.

Una de las posibles consecuencias del sufrimiento de importantes daños narcisistas en la infancia es la perversión. El perverso, de acuerdo a Sánchez, Gutiérrez, Rodríguez y Casado. (2008), puede conseguir el máximo grado de neutralización de la angustia si logra establecer con los objetos significativos un estatus; lo que significa, un modo de relación caracterizado esencialmente por la mentira, con el objetivo de que lo perverso pase a ser lo "normal", es decir, habitual y creador de norma. Se alcanza el estatus perverso cuando la perversión se disfraza de "ley" que, además es aceptada, incluso reclamada por el objeto.

Con respecto a la perversión diversos autores, entre estos Fernández (2008), explican la existencia de un "discurso perverso" mediante el cual la estructura perversa se expresa en el diálogo analítico y que se caracteriza por las distintas formas de la mentira, desde el juego de la mala fe hasta la mentira cínica. En base a esto, relacionan la hipótesis de que la estructura perversa no se puede enfocar tan sólo desde el ángulo del fetichismo, tampoco desde el narcisismo como perversión manifiesta, ni desde el sadismo, sino que este como tal requiere la articulación de estos tres factores determinantes, articulación variable ella misma según las diversas formas de la perversión, desde el extremo del fetichismo hasta el del gran sadismo o de la necrofilia. Subrayamos en la génesis de la perversión el papel de una familia "corruptora". Lo más frecuente es que sea la madre quien desempeñe el papel corruptor, pero también pueden desempeñarlo el padre o ambos padres. En esas familias reina un alto grado de ambigüedad acerca de la formulación y del cumplimiento de la ley paterna. De todos modos, la "perversión verdadera" no parece ser una opción estructurada por el sujeto en el intento de evitar la desintegración psicótica y de lidiar con la pulsión de muerte.

El estatus perverso, al igual que la perversión misma, tiene extrema dificultad para ser abordado en el psicoanálisis, por lo arcaico de la patología y por ser egosintónico. No obstante, la aproximación analítica, creo que debe estar orientada a poner de manifiesto la mentira fundamental, que es la síntesis y manifestación principal de las defensas en la perversión. 
Al momento de superar el Edipo el adolescente busca grupos y ya los padres no van a ser su modelo de identificación si no que los "compañeros", en cuanto al padre va a entrar en crisis puesto que siente la caída de su función como padre por lo que se ausentan y los hijos (adolescentes) sienten rechazo, pero el padre debe estar presente y acompañar a su hijo para que no ocurran las conductas desajustadas. Debido a que sintiendo un rechazo ya sea del padre o de la madre, buscan otras identificaciones en grupos, pandillas e incluso, plantean Inglés, Torregrosa, García., Martínez, Estévez y Delgado. (2014), siendo ellos los líderes de estos, por ende, la función del padre no se debe sólo a la etapa edípica, sino que también a la etapa de la adolescencia del hijo.

\section{Conclusiones}

Se determinó que las conductas desajustadas en los adolescentes son conductas que se van desarrollando a lo largo de la vida de los mismos, así que estas son producto de cómo se va estructurando su Yo y superyó y asimismo de su estructura clínica y aquello que lo conforma.

Las conductas desadaptadas de los adolescentes ante diversas situaciones, poseen una declinación de la metáfora paterna en la cual los sujetos por así denominados "conflictivos" son aquellos que no tienen un significante correcto de lo que es denominado como ley; de ahí la desviación de la metáfora paterna ya que no existe una figura a la cual imitar y si existe la misma no está clara para el sujeto; por lo cual la misma ocasiona problemas a niveles ya antes mencionados.

Las conductas desadaptadas normalmente se han vinculado con la personalidad, esta se forma por una combinación de sucesos internos y externos, con énfasis por supuesto en los internos, y por la capacidad de afrontamientos a estos sucesos. Un suceso importante, y que sin duda marca la personalidad de un sujeto es el de la metáfora paterna, por lo cual, se considera que estas conductas desadaptadas están ligadas en gran medida a como cada sujeto afronta esta metáfora paterna. 


\section{Referencias Bibliográficas}

Uribe, A. N, (2010). Adolescencia y ritos de transición. Una articulación del psicoanálisis postfreudiano y lacaniano, Colombia: Revista Electrónica de Psicología Social. Recuperado de: http://www.funlam.edu.co/revistas/index.php/poiesis/article/view/34

Negro, M. (2012). Estructura de la ley y del superyó, Colombia: Revista Affectio Societatis. Recuperado de: http://aprendeenlinea.udea.edu.co/revistas/index.php/affectiosocietatis/article/view/12045

Fernández, D. A. (2008). La importancia del padre en Psicoanálisis. Revista Internacional de Psicología, 9(02). Recuperado de: file://C:/Users/Usuario/Downloads/DialnetLaImportanciaDelPadreEnPsicoanalisis-6161373.pdf

Sánchez, M. M., Gutiérrez, R. B., Rodríguez, J. M., \& Casado, M. P. (2008). Influencia del contexto familiar en las conductas adolescentes. Ensayos: Revista de la Facultad de Educación de Albacete, (23), 391-408. Recuperado de: http://europa.sim.ucm.es/compludoc/AA?articuloId $=733488$

Inglés, C. J., Torregrosa, M. S., García-Fernández, J. M., Martínez-Monteagudo, M. C., Estévez, E., \& Delgado, B. (2014). Conducta agresiva e inteligencia emocional en la adolescencia. European Journal of Education and Psychology, 7(1). Recuperado de: http://www.redalyc.org/pdf/706/70624504006.pdf. 